Man Pann aub diefen Refultaten fdlieśn, Daß 0 b fafpeterfaure Silber aud im tročnen 3uftande ourd bas metallifbe Rupfer zerfezt werbe.

Beridftigung, bie Notiz einer kinittichen $\mathfrak{B i l}=$ Dung Der Bernjteinjáure betreffeno. $\quad B$, XX11. S. 165 dez 2Trdivis;

vom

2potbeer $\mathfrak{B}$ oaet tn Şeinbbera.

In obige Rotis bat fid ourd einen Drud : oder SAreibs fehler eine Untiatigeit in $\mathfrak{B e t r e f f}$ ber zu ben $\mathfrak{X e r f u d e n ~ a n s ~}$ gemanden Saure eingefdltaen, welde leidt zu Feblfdills fen Beranlaffung geben eonnte, baher id mid beeile, fels bige juberidtigen. Eb ift $n$ amtid nidt, wie angegeben, taus Wende Salpeterfáte, fonbern raudenbe Sarefel: $f \mathfrak{a}$ u $r$ fu ben $\mathfrak{X}$ erfuten angemand worben unb ourd bie: fe wurben jene Refultate erbalten.

\title{
Gynapin, ein neuez UlEaloio.
}

Die mit ber Peterfitie oft verwedifelt werbende Fqunos: gleife (Aethusa Cynapium) enthåt nad \$̧rofeffor $\mathfrak{F} i \mathrm{ci}$

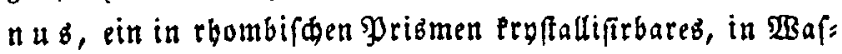
fer, Altohol, nidt in Aetber, lsstides Alfalois, 5nnapin. Das fowefelfaure Ennapin eryltallijirt in Y̧rigmeu (Kastner's Arch. XI. 144).

\section{(5y)odin.}

Seerr $\mathfrak{Y}$ rofeffor Semmola zu Reapel hat allb oer 2 urzel von Cynodon dactylon cine Subftanz erhalten, oie 
er Eynooin nennt. Darfellung: bie gefdalte $203 u r z e l$ wito aubgefodt, das ourd Sedimentiren erbaltente Defolt bis zur Syrupsoide yerdunftet, an einen eúblen Drt geftellt,

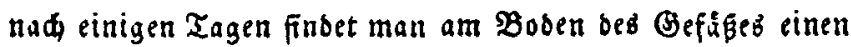
Eryfallinifden $\mathfrak{2} b$ 依, welder mit taltem $\mathfrak{B a f f e r}$ allöges wafden, in warmen Baffer aufgelsft und burd Umeryfrallifi:

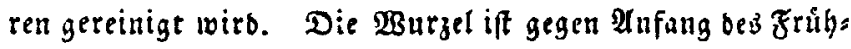
jahrs am reidhaltigften an dierem Stoff (l'Esculapio I. 43; Bullet. des sc. med. XII. 177).

Ueber cill in Den Samen von Impatieus Balsa-

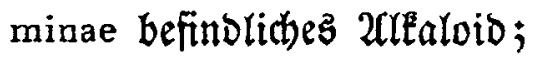

vom

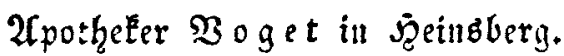

Fine vorláfige priffung ber Samen you Impatiens Balsaminea auf cin IItaloio entiprad meinen Errartungen.

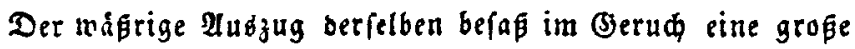

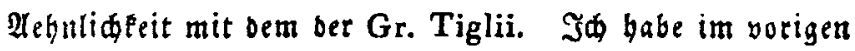
Şerbft 8-10 Dradmen der Samen gefammlet, werbe diefe getegentli弲 zu ferneren Berfuden-bermenden unb baráber nádftens ein Mebretes mittbeilen.

\section{Extract. Pulsatillae nigric.}

wito von 5 ferrn Şofrath Dr. Soa Riga febr gegen Sriábuften bei Rinoern empfoblen (Journ. der pract. Heilk. von Hufeland u. Osann. 1827. Aug. 122). 